\title{
Louis Châtellier, Philippe Martin (dirs.), L'écriture du croyant
}

Turnhout, Brepols, coll. «Bibliothèque de l'École des Hautes Études en Sciences Religieuses », 2005, 215 p.

\section{Bénédicte Sère}

\section{(2) OpenEdition}

Journals

Édition électronique

URL : http://journals.openedition.org/assr/5732

DOI : $10.4000 /$ assr. 5732

ISSN : $1777-5825$

Éditeur

Éditions de l'EHESS

Édition imprimée

Date de publication : 1 juin 2007

Pagination : 97-251

ISBN : 978-2-7132-2143-9

ISSN : 0335-5985

Référence électronique

Bénédicte Sère, «Louis Châtellier, Philippe Martin (dirs.), L'écriture du croyant », Archives de sciences sociales des religions [En ligne], 138 | avril - juin 2007, document 138-21, mis en ligne le 11 septembre 2007, consulté le 21 septembre 2020. URL : http://journals.openedition.org/assr/5732 ; DOI : https:// doi.org/10.4000/assr.5732

Ce document a été généré automatiquement le 21 septembre 2020.

(c) Archives de sciences sociales des religions 


\section{Louis Châtellier, Philippe Martin (dirs.), L'écriture du croyant}

Turnhout, Brepols, coll. «Bibliothèque de l'École des Hautes Études en Sciences Religieuses », 2005, 215 p.

\section{Bénédicte Sère}

1 Introduit par Louis Châtellier et Philippe Martin, conclu par Jean Le Brun, ce recueil de seize articles est le produit de plusieurs rencontres et colloques organisés à Nancy et Strasbourg par l'équipe « Esprit moderne en religion» (GDR 2342) dont l'un des axes de recherche prioritaires concerne l'individu en religion. L'ambition de départ est claire : les études de cas cherchent à s'approcher le plus possible du vécu religieux des hommes du temps; il s'agit de débusquer les individus dans leur acte d'écriture pour tenter de sentir l'épaisseur de leur croyance, les fluctuations de leur foi et autres vicissitudes de leur vie spirituelle. D'emblée, les auteurs l'annoncent : c'est l'acte d'écriture plus que le contenu même de l'écrit qui atteste une expérience spirituelle propre, que celle-ci soit singulière ou conformiste, fragile ou sans heurt, modérée ou fervente.

2 La matière des analyses part de cas précis et détaillés. Nombre d'exemples étudiés sont centrés sur les espaces géographiques lorrain et alsacien, espaces bien maîtrisés par les auteurs du volume, pour la plupart professeurs à l'université de Nancy II ou à l'université Marc Bloch-Strasbourg II. Il s'agit ici de la figure d'un missionnaire lorrain, Augustin Schoeffler, redécouvert à travers ses lettres (Bernard Stelly); on note là l'exemple de chanoinesses de quatre chapitres lorrains (Françoise Boquillon); c'est aussi la double narration d'un moine bénédictin de Saint-Mihiel (Jean Lanher) ou encore l'essai de reconstitution autobiographique de Martin Bucer, le réformateur de Strasbourg (Annie Noblesse-Rocher) et quelques figures de pasteurs luthériens d'Alsace et de Moselle au xIX siècle (Matthieu Arnold). Pour autant, l'ouverture à d'autres régions ne manque pas: sont évoqués les Cévennes, cette autre terre de la cohabitation confessionnelle (Robert Sauzet), le Berry (Didier Boisson), l'Aveyron (Ludovic Viallet), Reims (Stefano Simiz), Cavaillon (Frédéric Meyer) ou encore Trêves (Bernhard Schneider), la Croatie habsbourgeoise (Luc Oreskovic) et le Liban maronite (Bernard Heyberger). De même, si les exemples se concentrent sur les $\mathrm{xVI}^{e}$ et $\mathrm{xVII}^{\mathrm{e}}$ siècles, 
l'ampleur chronologique dépasse les bornes traditionnelles dévolues à l'époque moderne puisque les études de cas remontent au XIV ${ }^{e}$ siècle et se prolongent jusqu'au XIX ${ }^{e}$ siècle. Par-delà cette amplitude spatio-temporelle, ce sont les sources écrites qui témoignent, dans ce recueil, de la plus grande diversité : les responsables du volume ont choisi de les organiser autour de trois pôles pour en unifier au mieux la présentation. Un premier pôle concerne la correspondance, genre incontournable de l'écriture spirituelle, plus ou moins codé selon le destinataire, le contexte d'émission, la publicité de la diffusion, la réutilisation politique. À travers la correspondance des membres de quatre compagnies de la Propagation de la foi entre 1647 et 1685, Catherine Martin reconstitue la vitalité des réseaux dévots au XvII siècle, à travers la qualité des relations qui lient les membres à leur fondateur, et l'intensité de leur activité. Dans un autre contexte, Luc Oreskovic présente les lettres de deux aristocrates condamnés à mort par l'empereur d'Autriche pour compromission avec les Ottomans : ces lettres révèlent leur idéal baroque de piété, idéal vraisemblablement travaillé par des auteurs qui se savaient lus par l'administration impériale. La correspondance d'André-Marie Ampère et celle d'Augustin Schoeffler offrent à l'historien une source complémentaire pour revisiter ou découvrir l'authentique physionomie spirituelle de ces deux hommes, masquée par des sources plus hagiographiques. La seconde partie du volume regroupe des contributions sans lien du point de vue des sources étudiées: actes notariés, journalier d'un maître charpentier, livre de raison d'un chanoine, mémoires et testaments de chanoinesses, enfin marginalia de livres de dévotion. Cette partie, composée de varia, a pour ambition de fouiller « les recoins les plus cachés » de cette écriture du croyant aux modalités si diverses. Il s'agit, pour Ludovic Viallet, de scruter les discrètes invocations religieuses hors-formulaires laissées dans leurs actes par certains notaires aveyronnais et ardéchois: elles traduisent une conception sacralisée de l'écriture notariée, investie d'une mission quasi divine dans la parole à conserver. Le journalier de Jehan Pussot et le livre de raison de Jean-Gaspart de Grasse restituent tous deux la vision du monde et les évolutions religieuses de deux hommes dans le contexte des mutations profondes engendrées par la réforme catholique. Le premier témoignage transcrit la perplexité d'un homme déstabilisé par les évolutions rapides, notamment liturgiques et pastorales. L'autre rend compte d'une religiosité parfaitement pondérée, ni dévote, ni janséniste, loin de la ferveur partisane des uns ou de la crédulité naïve des autres. Certains écrits de chanoinesses sont l'expression d'une piété sincère aux formes proches des dévotions populaires. Quant aux notes de lecture étudiées par Philippe Martin dans la littérature dévote des XVIII ${ }^{\mathrm{e}}$ et $\mathrm{XIX}^{\mathrm{e}}$ siècles, elles témoignent d'un véritable acte d'appropriation du livre, lieu d'une lecture active et d'une écriture engagée du croyant devant Dieu. Le troisième chemin que prend la plume du croyant touche aux récits de vie. Écrire est, dans ce contexte, une occasion de relire sa vie, un besoin d'y mettre du sens, d'y trouver une direction, un désir d'y voir un progrès spirituel. Dom Loupvent narre à deux reprises son pèlerinage à Jérusalem : à onze ans d'intervalle entre les deux rédactions, on y lit le même homme mais non le même croyant. Quand Martin Bucer écrit ses textes apologétiques, il se défend des accusations portées contre lui (moine défroqué et marié) mais surtout contribue à préciser les points de la nouvelle théologie réformée concernant la vanité de l'état monastique et la valeur de la chasteté dans l'état de mariage. Charles charlot d'Argenteuil, curé catholique converti au protestantisme, retrace l'histoire de sa conversion en écrivant à ses anciens paroissiens : il relit dans sa vie passée tous les ancrages du doute qui l'ont conduit à poser son choix. Enfin, ce sont les figures d'une 
mystique libanaise, de trois pasteurs alsaciens et deux prélats allemands qui sont présentés et dont le récit autobiographique offre des éléments pour retracer un cheminement spirituel aux prises avec les réalités politiques d'une époque.

3 Pour les auteurs du volume, l'acte d'écriture est donc bien ce qui unifie les contributions. Le fait de l'écriture est plus révélateur que le contenu lui-même. Parce qu'elle touche au problème si intangible de la foi, l'écriture du croyant se révèle particulièrement délicate comme témoignage historique. Elle est cette expérience propre à travers laquelle l'historien cherche à entendre l'individu derrière les stéréotypes et les conformismes. Dans le Mémorial d'André-Marie Ampère, il lit un homme en quête d'intelligence de sa foi, une foi fragile soumise aux doutes et aux interrogations, dans un contexte où l'Église répondant difficilement aux exigences de ces intellectuels, pouvait elle-même générer cette situation d'instabilité et d'inconfort spirituels. La correspondance du grand savant guillotiné en 1793 permet ainsi à l'historien de découvrir les vicissitudes de cet itinéraire spirituel que masquait le discours hagiographique plus connu. De même, les deux versions du pèlerinage à Jérusalem, écrites par Dom Loupvent en 1532, d'une part, et en 1543, d'autre part, montrent par leurs infimes inflexions l'évolution de la croyance à la foi dans le sens d'un affermissement de ses adhésions, débarrassées des légendes et du merveilleux. Ailleurs, ce sera la tonalité propre d'une piété que l'historien pourra reconstituer: piété baroque des condamnés croates, sensibilité humaniste de tel chanoine, ferveur mystique de telle religieuse.

4 Le volume insiste donc sur l'écriture comme expérience spirituelle : produit d'une injonction, d'un désir ou d'une nécessité, l'écriture du croyant actualise en même temps qu'elle l'élucide la foi qu'elle transcrit. L'historien envisage à travers cette expérience une trace vivante qu'il se doit d'interpréter dans une ouverture infinie des interprétations à la manière de l'interprétation de l'Écriture elle-même. Par là même, tout «écrit » du passé, envisagé sous l'angle de son acte propre, est objet d'histoire. C'est la leçon que nous offrent les auteurs de ce volume nous invitant à appliquer leurs résultats aux autres périodes et champs de l'histoire. Une question cependant demeure. Assurément, le titre du recueil est suggestif ; la conclusion de Jacques Le Brun qui en développe la teneur est brillante. Pour autant, n'est-ce pas très simplement l'objet de toute étude historique de rechercher derrière la source écrite le visage de l'individu et l'épaisseur de son vécu - religieux ou autre? 